\title{
A Study on the Effect of Visual Feedback Training Combined with Orthodox Training on the Balancing Function in Hemiplegic Patients Caused by Stroke
}

\author{
Yu Yang ${ }^{1, a, *}$, Wang Haijun ${ }^{1, b}$, Wang Yongjian ${ }^{1, c}$, Ling Yan ${ }^{2, d}$, Sun Liang,e \\ ${ }^{1}$ First Affiliated Hospital of Qiqihar Medical University, Qiqihar Pukui Street, Qiqihar, China \\ ${ }^{2}$ Hangzhou West Lake Juyong Eye Hospital, Hangzhou Wener Street, Hangzhou, China \\ a wenzhang4477@163.com, ${ }^{\mathrm{b}}$ zxm86000@163.com, ${ }^{\mathrm{c}}$ 396171469@qq.com, ${ }^{\mathrm{d}}$ \\ fanchuanlo@qq.com ${ }^{\mathrm{e}} 1823777172 @ q q . c o m$ \\ *corresponding author
}

Keywords: Mindfulness training, Visual feedback, Stroke, Rehabilitation, Balance function.

\begin{abstract}
The purpose of this study is to explore the effect of psychological training on the daily life ability of stroke patients with hemiplegia, and to provide a guiding method for the rehabilitation of these patients. A total of 72 patients with stroke were randomly divided into experimental group $(\mathrm{n}=36)$ and control group $(\mathrm{n}=36)$. The experimental group was trained with visual feedback training and daily training, and the control group was trained according to routine visual balance and daily nursing. There were significant differences in the evaluation indexes between the two groups before treatment, and the improvement of BBS score, track length and peripheral area score in the experimental group were significantly higher than that in the control group $(\mathrm{P}<0.05)$. The combination of clinical rehabilitation training and psychological training has improved the ability of daily life of patients and promoted the therapeutic effect. $(\mathrm{P}>0.05)$. After 8 weeks of treatment, the above indexes were improved in both groups $(\mathrm{P}<0.05)$ compared with the control group.
\end{abstract}

\section{Introduction}

Stroke is one of the more common chronic diseases in our country. Most of the patients left limb dysfunction after stroke. Only half a year later, only about one-third of the patients recovered the dexterity of the affected limbs ${ }^{[1]}$. Balanced dysfunction is one of the common functional problems of stroke or other patients after craniocerebral injury. Therefore, seriously affect the patient's rehabilitation and quality of life. As the visual system is to provide information about the surrounding environment and body movement and direction, the balance between the balance function and the visual system is equally important. Various methods are more and more applied to the evaluation and training of the balance function. The assessment of balanced function and targeted training of stroke patients with hemiplegia are of great significance.

Mindfulness training is referred to the various psychological therapy with the heart of mindfulness ${ }^{[2]}$, the current more mature mindfulness treatment, including righteousness cognitive therapy, acceptance and commitment therapy, righteousness decompression therapy and dialectical behavioral therapy. This study uses mindful cognitive therapy, is to observe the things themselves our thoughts, emotions, physical feelings and the surroundings. Mindfulness tells us that the world is a mirror of reflection: clear, just, no difference. When practicing mindfulness, we can perceive and realize that the scene of life is happening without being stumbled into it and totally ignorant. Mindfulness training is widely used in the treatment of anxiety, depression, compulsive, impulsive and other emotional psychological problems, personality disorders, interpersonal communication, addiction and other aspects of treatment also has a large number of applications. Not only that, medical research also shows that it is helpful to practice some types of mindfulness exercises in improving cardiovascular system problems, enhancing immunity, relieving pain (such as neuropathic headache, low back pain, etc. ${ }^{[3]}$. In this paper, the visual balance feedback training instrument with the mindfulness training were applied to assess stroke patients with hemiplegia, 
through observing the static balance function under the conditions with open and closed eyes to explore the visual system to promote the balance of the potential recovery of functional recovery after the balance function was destroyed, and observed its clinical efficacy.

\section{Object and methods}

\subsection{Object}

A total of 45 males and 27 females with an average age of $59.7 \pm 8.2$ years were selected. The criteria for admission were ${ }^{[4]}$ : (1) Select the patients with first brain Infarction, cerebral hemorrhage; (2) through the head CT or MRI examination, the patients have been diagnosed as unilateral lesions, the condition is relatively stable; (3) duration of 12 weeks; (4) in addition to motor dysfunction, Visual and cognitive function and understanding of the obstacles; (5) the patients can keep the legs standing for at least 60 seconds. Patients were randomly divided into experimental group and control group, both were 36 cases.As followed is Table 1 .

Table 1. Experimental group and control group general data statistics

\begin{tabular}{|c|c|c|c|c|c|c|c|c|}
\hline \multirow{2}{*}{ Group } & \multicolumn{2}{|c|}{ Sex Average age } & \multicolumn{2}{|c|}{ Average duration (Days) } & \multirow{2}{*}{\multicolumn{2}{|c|}{$\begin{array}{l}\text { Lesion site (cases) } \\
\text { Cerebral hemorrhage }\end{array}$}} & \multirow{2}{*}{\multicolumn{2}{|c|}{$\begin{array}{l}\text { Lesion cause (cases) } \\
\text { cerebral infraction }\end{array}$}} \\
\hline & Male & Female & Left & Right & & & & \\
\hline EXP. & 22 & 14 & $58.32 \pm 11.43$ & $44.26 \pm 9.47$ & 25 & 11 & 25 & 11 \\
\hline Con. & 20 & 16 & $56.18 \pm 10.51$ & $42.83 \pm 8.66$ & 21 & 15 & 22 & 14 \\
\hline
\end{tabular}

\subsection{Methods}

The experimental group using visual feedback training and correct training to carry out rehabilitation training, training every day shall not be less than half an hour; the control group using the visual feedback training instrument for rehabilitation training according to their own situation, training every day shall not be less than half an hour. The training group and the control group were required to be carried out separately in the open eyes and closed eyes. First, the balanced training in both groups were taken out at the same time, coordinated the development of large and small size muscles, improve the body feeling. Through the evaluation results of patients reflected by the balanced training instrument, we selected the appropriate model for training, according to the target on the display, they can complete the conversion of their own center of gravity. They were trained once by the professional instructors every day, according to their own adaptation, the time shall not less than 30 minutes. Mindfulness training methods are mainly divided into three minutes breathing space and righteousness walking. Three minutes breathing is to allow patients to use sitting, close your eyes to experience the current ideas and emotions and perception of the body as a whole awareness. This practice allows patients to experience the pain caused by adverse reactions to establish a new relationship for the lifestyle to bring a fundamental change; Righteous walking makes patients breath naturally, without control, and pay attention to walking itself or experience the touch between the ground and the foot, as well as the overall movement of the body and the gravity perception of the body center movement, each time shall not less than 10 minutes, the main theme emphasizes the patients simply live in the moment, concern about this step, do not need to do anything or go anywhere or compete for anything.

\subsubsection{Experimental apparatus}

Using the German Dr-wolff balance test and training instrument, Balance-check can be used for dynamic balance testing and training, applied to functional rehabilitation training, restore the activity and strength of injured joints, coordinate the development of large and small muscles, improve the body feeling, to prevent sports injuries, also improve the balance ability of the elderly to prevent falls. Because of its simple operation, the test and training forms are flexible, so it is very interesting and suitable for functional assessment and nursing rehabilitation training. 


\subsubsection{Test mode}

Test patterns are diverse, according to the situation of subjects, the difficulty levels, test time and manners are set. The results are given after the test is completed. Training ways are in a variety and full of fun. You can set the training difficulty level, training time and ways according to the preferences of the subjects. During training, the subjects move through the center of gravity, and complete the specified tasks as soon as possible, auditory feedback makes training more interesting.

\subsubsection{Assessment methods}

The experimental group and the control group were respectively evaluated by the balance stabilizer after 8 weeks of training. The evaluation index was the trajectory and the peripheral area of the open and closed eyes. The smaller the trajectory value, the better the stability of the patient; the smaller the peripheral area value, the better the stability of the patient ${ }^{[5]}$.

Berg balance scale (BBS) is one of the ways mainly used to assess the degree of balance dysfunction. Before the formal test, the patients were allowed to practice 1 or 2 times, in order to ensure the stability of the test process, all tests were completed by the same therapist. The lowest score of each item is zero, the highest is four and the total is 56 points, the high score means the balance condition is good, if the score below 40 points, suggesting a risk of falling.

\subsection{Statistical methods}

SPSS18.0 statistical software were used for statistical analysis, compared using the variance analysis, with $\mathrm{P}<0.05$ for a significant difference.

\section{Results}

\subsection{BBS assessment}

There was no significant difference in BBS scores between the experimental group and the control group before the training,the scores of both were significantly increased and the results of the experimental group were significant $(\mathrm{P}<0.01)$, the results showed that the rehabilitation patients who used the combination of visual feedback and mindfulness training were significantly better than the patients who simply used visual balance training . The result is shown in Table 2.

Table 2. Experimental group and control group before and after training BBS score $(\mathrm{x} \pm \mathrm{s})$

\begin{tabular}{|l|l|l|l|}
\hline Group & Cases & \multicolumn{1}{|c|}{ BBS1 } & \multicolumn{1}{c|}{ BBS2 } \\
\hline Experimental group & 36 & $28.46 \pm 4.38$ & $39.73 \pm 4.19$ \\
\hline Control group & 36 & $27.81 \pm 3.69$ & $32.45 \pm 3.75$ \\
\hline P value & & 0.79 & 0.00 \\
\hline
\end{tabular}

Note: BBS1 indicates that Berg score before training; BBS2 means Berg score after training; Before training, comparing the experimental group and control group, $\mathrm{P}>0.05$; after training, $\mathrm{P}<0.05$.

\subsection{The determination of length of the track and the outer area with open eyes and closed eyes}

There was no significant difference in trajectory length and peripheral area between the experimental group and the control group before training. Both groups were improved, which was statistically significant $(\mathrm{P}<0.001)$, the experimental group was more significant than the control group $(\mathrm{P}<0.05)$. 
Table 3. Comparison of the length of the track and the outer area with open and closed eyes of experimental and control groups with biped standing $(\mathrm{x} \pm \mathrm{s})$

\begin{tabular}{|c|c|c|c|c|c|c|c|c|c|}
\hline \multirow{2}{*}{ Group } & \multirow{2}{*}{ Cases } & \multicolumn{2}{|c|}{ Length B.T(mm) } & \multirow{2}{*}{$\begin{array}{c}\text { Length } \\
\text { Open } \\
\end{array}$} & \multirow{2}{*}{$\frac{\text { A.T }(\mathrm{mm})}{\text { Closed }}$} & \multicolumn{2}{|c|}{ Outer area B.T(mm) } & \multicolumn{2}{|c|}{ Outer area A.T(mm) } \\
\hline & & Open & Closed & & & Open & Closed & Open & Closed \\
\hline Exp. & 36 & $\begin{array}{l}656.14 \pm \\
181.34\end{array}$ & $\begin{array}{l}1146.21 \pm \\
294.56\end{array}$ & $\begin{array}{l}347.28 \pm \\
143.42\end{array}$ & $\begin{array}{l}624.32 \pm \\
281.59\end{array}$ & $\begin{array}{l}1348.22 \pm \\
871.54\end{array}$ & $\begin{array}{l}3120.27 \pm \\
1471.18\end{array}$ & $\begin{array}{l}605.16 \pm \\
579.41\end{array}$ & $\begin{array}{l}1319.43 \pm \\
769.75\end{array}$ \\
\hline Con. & 36 & $\begin{array}{l}619.34 \pm \\
145.16\end{array}$ & $\begin{array}{l}911.24 \pm \\
273.23\end{array}$ & $\begin{array}{l}484.72 \pm \\
143.91\end{array}$ & $\begin{array}{l}726.82 \pm \\
227.84\end{array}$ & $\begin{array}{l}1137.24 \pm \\
776.31\end{array}$ & $\begin{array}{l}2674.52 \pm \\
1156.17\end{array}$ & $\begin{array}{l}981.43 \pm \\
628.79\end{array}$ & $\begin{array}{l}2161.85 \pm \\
886.3\end{array}$ \\
\hline P Value & & 0.71 & 0.22 & 0.00 & 0.29 & 0.64 & 0.49 & 0.18 & 0.03 \\
\hline
\end{tabular}

Note: Comparison between the two groups before training $\mathrm{P}>0.05$

Comparison between the two groups after training $\mathrm{P}<0.05$

\section{Discussion}

With the widespread use of electronic technology in the medical field, the emergence of the balance function training instrument provides a new way for the assessment and training of hemiplegic patients in terms of balance function. It is a combination of visual feedback and the integration of various components of human balance training system. This instrument has a high balance of stability, high reliability, and has been used in the clinical efficacy ${ }^{[6]}$. Balancing ability refers to the ability to maintain the body's center of gravity on the support surface within the stability limits, and the center of gravity of the body must be reasonably distributed, symmetrical, and maintained at any reasonable balance symmetrical distribution. In this study, the length of the track and the outer area with open and closed eyes of experimental and control groups with biped standing showed in the Table 3 study results suggested the gravity center condition of the experimental group and the control group of open eyes and eyes closed. Before the training, there was no difference ( $\mathrm{P}>0.05)$. After the training, there was a significant increase between the two groups $(\mathrm{P}<0.05)$, indicating that the visual plays a role in maintaining the distribution of the center of gravity of the human body. Balanced dysfunction is one of the common dysfunctions of patients after stroke or craniocerebral injury. Stroke hemiplegia patients with central nervous system or peripheral nervous system vulnerable to damage caused by other system functions are also easy to change, and thus easily lead to balance dysfunction, is the patient's daily life, walking ability of one of the basis ${ }^{[7,8]}$. In this study, the experimental group of patients with open eyes when the long trajectory and closed eyes when the peripheral area after treatment than the control group was significantly improved. Although the outer area of the open eyes and the length of track of closed eyes after treatment are less than before treatment, indicating that the body swing amplitude decreases, stability increasing. Another factor in maintaining balance is the input of the appropriate sensory system, which in the case of stability and instability, the visual, somatic sensations and vestibular sensations play a different role. Therefore, in the case of visual loss, the balance of capacity have declined. The results of this study show that both before and after treatment, the balance of open eyes are better than closed eyes.

The combination of mind training and visual feedback balance training will help to further improve the overall recovery of stroke patients with hemiplegia. Mindfulness training is through the whole body to focus on their own experience, is a kind of not disturbed, sober conscious state given to patients with certain assurance support to help patients establish an effective coping style. Through this way of training can promote individual adaptation and better response to the environment, reduce bad mood, improve the positive life satisfaction, self-confidence, optimism and so on.

\section{Conclusion}

This study is mainly through visual feedback balance training combined with mind training, to promote the recovery of brain function as soon as possible. From the results of the study can be seen in the balance of the function after the injury is dependent on the adjustment of visual function 
to maintain the balance of people, through the visual training to adjust the proportion of maintaining a high balance, relying on the visual system to help training can strengthen the application of visual feedback training It is possible to promote the early recovery of its balance function. Through these two training methods at the same time, can promote each other, interact with each other to improve the treatment of patients with a certain clinical significance.

\section{Acknowledgements}

Fund project: Qiqihar science and technology plan project(Nomber:SFZD-2015004)

\section{References}

[1] Zhang QiuMei,Gao ChunHua.(2014)Effects of motor visual feedback training on upper limb function in stroke patients with hemiplegia.Chinese Journal of physical medicine and rehabilitation, 36(4),278-280.

[2] Wang GuoLi,Li JianMin,Zhao YaNing.(2014)Observation on rehabilitation effect of patients applying meditation training for Shoulder handsyndrome after. China Journal industrial medicine, 17(12), 1902-1904.

[3] LiuYue,SuLiMei,xieShuShan.(2013)Treatment of shoulder hand syndrome with stroke combined with rehabilitation training in stroke stage I.Chinese Journal of rehabilitation medicine,28 (3), 267-268.

[4] Chinese society of neuroscience, Chinese society of Department of neurosurgery. (1996) Keypoints indiagnosis of cerebrovascular diseases.Chinese department of neurology medicine, 29 (6),379.

[5] Yang Ting, Gao Zheng, Yin YuWen.(2012)Clinical study on the effect of visual feedback balance training apparatus for the balance function of stroke patients.Chinese Journal of clinicians, 6(19),6046-6048.

[6] Wang Sheng,YangJu,Zhu Yi.(2011)Reliability and validity of balanced feedback training apparatus for static balance test of hemiplegic patients with brain injury.Chinese Journal of rehabili--tation medicine, 26,1035-1038.

[7] HuangXiaoJing,DouZuLin,QiuWeiHong.(2011)Effect of dynamic posture balance training on balance function in stroke patients with hemiplegia.Chinese Journal of rehabilitation medicine, 26, 1029-1034.

[8] Duncan P W, Zorowitz R, Bates B, et al. (2005)Management of adult stroke rehabilitation care. Stroke, 36(9),100-14 